\title{
On the Stability Analysis of Thin Walled Shell Structures Containing Gas or Fluid
}

Marc Haßler and Karl Schweizerhof

Universität Karlsruhe, Institut für Mechanik

2006

Institut für Mechanik

Kaiserstr. 12, Geb. 20.30

76128 Karlsruhe

Tel.: +49 (0) 721/ 608-2071

Fax: +49 (0) 721/ 608-7990

E-Mail: ifm@uni-karlsruhe.de

www.ifm.uni-karlsruhe.de 


\title{
ON THE STABILITY ANALYSIS OF THIN WALLED SHELL STRUCTURES CONTAINING GAS OR FLUID
}

\author{
Marc Haßler $^{1}$ and Karl Schweizerhof ${ }^{2}$ \\ ${ }^{1}$ Institut für Mechanik, Universität Karlsruhe (TH) \\ Englerstraße 2, D-76131 Karlsruhe, Germany \\ e-mail: Marc.Hassler@ifm.uni-karlsruhe.de \\ 2 Institut für Mechanik \\ e-mail: Karl.Schweizerhof@ifm.uni-karlsruhe.de
}

Keywords: Fluid-Structure Interaction, Stability, Large Deformations, Finite Elements.

\begin{abstract}
Thin shell or membrane structures containing gas or fluid are widely standard, such as oil and water tanks, gas containers or even atmospheric balloons, pressurized girders or inflatable dams. For such thin walled structures the gas or fluid can be considered either as support or as loading. It may have a major influence on the stability behavior under other external loading as for example in the Tensairity-concept [3], where internal air pressure in combination with some external strengthening is used to overcome buckling of thin walled girders. The goal of this contribution is to present some investigation of the influence of such a gas or fluid support on the stability, here the eigenvalues and eigenmodes of the stiffness matrix of shell or membrane-like structures undergoing large displacements. For this purpose an analytical meshfree or lumped parameter description for the fluid/gas (see also [1]], [7], [8] and [9]) is taken, which yields a special structure of the nonlinear equations representing the change of the gas or fluid volume or alternatively the change of the wetted part of the shell surface. Finally this procedure leads first to the so-called load-stiffness matrix [11], to which several rank-one updates depending on the volume containing either gas or fluid or both are added. These rank updates are a key part in the stability analysis: They describe the different coupling of the fluid or gas volume change with the structural displacements in addition to the deformation dependence of the standard pressure. The specific rank-one updates allow the derivation of a very efficient algorithm to compute the modifications of the eigenvalues and eigenmodes of the original stiffness matrix without gas or fluid loading or support.
\end{abstract}




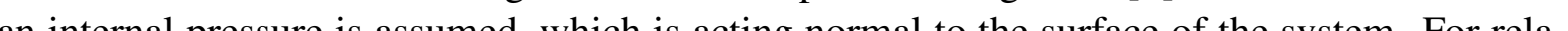
tively small deformations and structures not prone to instability this approximation is adequate. Howewer, for large deformations (see also [10], [11]) and stability problems further effects have to be considered, especially the change of the volume and the inner state variables of the gas and/or the fluid. The fact that the applied gas or fluid pressure is volume dependent is often neglected as also for example the volume dependence of the current fluid level.

This contribution is devoted to the investigations of the influence of such a fluid/gas support or loading on the eigenvalues and eigenmodes of the stiffness matrix of thin walled shell structures undergoing large displacements. For this purpose we refer to the former contributions of the authors group [7], [8] and [9] and the works of Bonet et al. [1], which dealt with gas and fluid supported shell structures and considered both the shape and volume dependence of the hydrostatic pressure distribution. With these derivations it is possible to come up with an analytical formulation of the fluid/gas only described by surface integrals over the surrounding wetted structure. Hence no FE discretization of the fluid or the gas is necessary. A further contribution of the authors group currently being in progress [4] merges all these results and thus provides a unified formulation for closed chambers containing any combination of volume dependent loading. Therefore the notations in the current contribution were adapted from [4].

First the necessary governing equations of the problem will be briefly derived via their virtual work expressions. In a further step the obtained variation of the gas/fluid potential has to be linearized for a Newton-type solution algorithm. After FE-dicretization of the linearized weak form a stiffness matrix updated by several dyadic products is obtained, which reflects the volume dependence of the inner state variables. These specific rank updates are a main part in the stability investigation, because they allow the derivation of a very efficient algorithm to determine the influence of the fluid or gas loading on the eigenvalues and eigenmodes of the stiffness matrix. The numerical examples contain large deformation analyses of fluid and gas filled shell structures with rather thin flexible walls and illustrate the performance of the derived algorithm.

\section{Governing Equations}

The general problem of thin walled shells containing gas and/or fluid (see figure 1) is prescribed by its properties of the potential energy $\Pi$, which consists of the - here for simplicity - elastic potential $\Pi^{e l}$ of the surrounding structure of the containment, the potential $\Pi^{f s i}$ of the internal fluid and gas and the work $W^{e x}$ of the external forces. For equilibrium the variation of the total potential energy then vanishes identically.

\subsection{Virtual Work Expression}

The variation $\delta \Pi$ of the total potential energy is given by the variation of its parts:

$$
\delta \Pi=\delta \Pi^{e l}+\delta \Pi^{f s i}-\delta W^{e x}=0
$$

For the elastic energy and the work of the standard external loading, such as e.g. dead loading, we refer to standard FE-text books [13], [15]. In the following the focus is only on the potential 
$\boldsymbol{n}^{f}$ and $\boldsymbol{n}^{o}$ the non-normalized normal vectors on the fluid boundary and on the fluid level. $\boldsymbol{x}^{f}$ and $\boldsymbol{x}^{o}$ define the position vectors to a wetted structural point and to the fluid surface. $\delta \boldsymbol{u}^{g}$, $\delta \boldsymbol{u}^{f}$ and $\delta \boldsymbol{u}^{o}$ denote the virtual displacement on the particular boundary.

\subsubsection{Incompressible fluid with free fluid surface and additional gas loading}

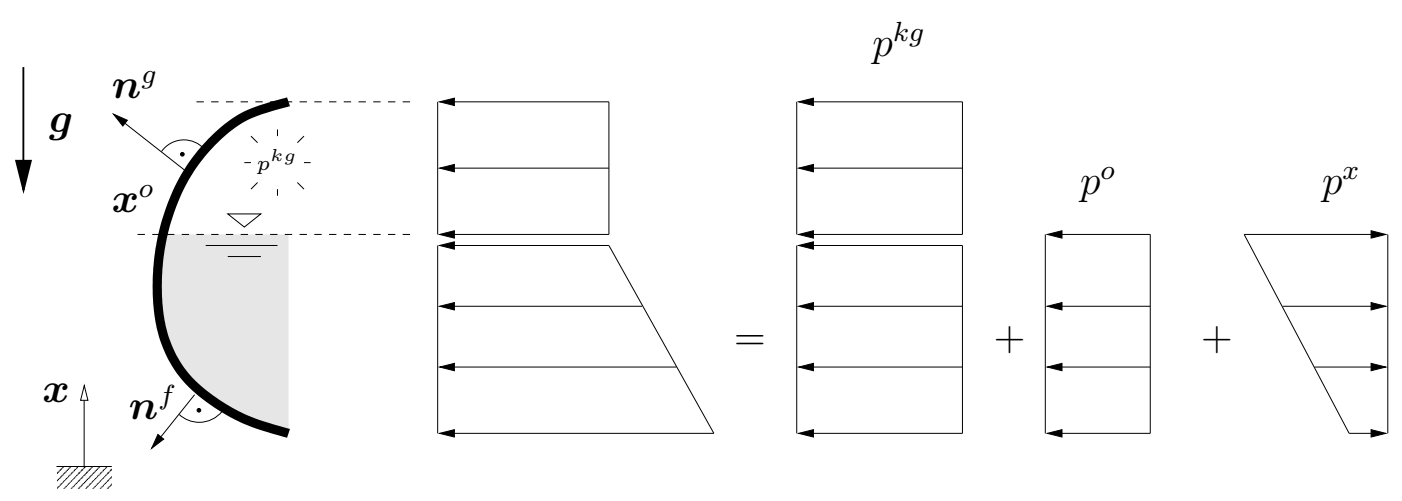

Figure 2: Hydrostatic pressure distribution in a chamber filled with gas and an incompressible heavy fluid

The gas and fluid pressure distribution for this case is depicted in figure 2. Using equations (7) and (8) in (2) gives us along with the pressures

$$
\begin{gathered}
p^{x}=\rho \boldsymbol{g} \cdot \boldsymbol{x} \\
p^{o}=\rho \boldsymbol{g} \cdot \boldsymbol{x}^{o} \\
p^{f}=p^{o}-p^{x}-p^{k g}
\end{gathered}
$$

the virtual gas and fluid potential for an incompressible fluid with free fluid surface and additional gas loading

$$
\delta \Pi^{f s i}=\int_{\eta^{f}} \int_{\xi^{f}} p^{f} \boldsymbol{n}^{f} \cdot \delta \boldsymbol{u}^{f} d \xi d \eta-\int_{\eta^{g}} \int_{\xi^{g}} p^{k g} \boldsymbol{n}^{g} \cdot \delta \boldsymbol{u}^{g} d \xi d \eta .
$$

\section{Linearization}

The virtual expression (12) is now written as a Taylor series expansion up to the first order term in order to embed it into a numerical solution procedure, e.g. in a Newton-type scheme. A consequent linearization of the virtual work (12) at a given state $t$ then leads to

$$
\delta \Pi_{l i n}^{f s i}=\int_{\eta^{f}}^{\bullet \text { Residual }} \int_{\xi^{f}} p_{t}^{f} \boldsymbol{n}_{t}^{f} \cdot \delta \boldsymbol{u}^{f} d \xi d \eta+\int_{\eta^{g}} \int_{\xi^{g}} p_{t}^{k g} \boldsymbol{n}_{t}^{g} \cdot \delta \boldsymbol{u}^{g} d \xi d \eta
$$

- Pressure change parts 


$$
+\quad \int_{\eta^{f}} \int_{\xi^{f}} p_{t}^{f} \Delta \boldsymbol{n}^{f} \cdot \delta \boldsymbol{u}^{f} d \xi d \eta+\int_{\eta^{g}} \int_{\xi^{g}} p_{t}^{k g} \Delta \boldsymbol{n}^{g} \cdot \delta \boldsymbol{u}^{g} d \xi d \eta
$$

\subsection{Incremental pressure changes}

The incremental pressure changes can also be directly adapted from [9], [4]. The mass conservation of the fluid and the adiabatic state equation of an ideal gas with e.g an isentropic exponent $\kappa=1.4$ then lead to:

$$
\begin{gathered}
\Delta p^{k g}=-\alpha_{t} \int_{\eta^{g}} \int_{\xi^{g}} \boldsymbol{n}_{t}^{g} \cdot \Delta \boldsymbol{u}^{g} d \xi d \eta-\alpha_{t} \int_{\eta^{f}} \int_{\xi^{f}} \boldsymbol{n}_{t}^{f} \cdot \Delta \boldsymbol{u}^{f} d \xi d \eta, \quad \text { with } \quad \alpha_{t}=\kappa \frac{p^{k g}}{\bar{v}^{g}} \\
\Delta p^{o}=-\gamma_{t} \int_{\eta^{f}} \int_{\xi^{f}} \boldsymbol{n}_{t}^{f} \cdot \Delta \boldsymbol{u}^{f} d \xi d \eta, \quad \text { with } \quad \gamma_{t}=-\rho_{t}\left(\int_{\eta^{f}} \int_{\xi^{f}} \boldsymbol{n}_{t}^{f} \cdot \boldsymbol{g} d \xi d \eta\right)^{-1} \\
\Delta p^{x}=\rho_{t} \boldsymbol{g} \cdot \Delta \boldsymbol{u}^{f}
\end{gathered}
$$

\subsection{Normal change parts}

The normal change parts for the gas and fluid domains can be adapted from [7] and [8]. For the fluid domain the additional part due to the non-constant pressure distribution $p_{t}^{x}$ must be considered. By introducing the skewsymmetric tensors (see also [10] and [5])

$$
\begin{aligned}
& \boldsymbol{W}^{\bar{\xi}}=\boldsymbol{n}_{t} \otimes \boldsymbol{x}^{\bar{\xi}}-\boldsymbol{x}^{\bar{\xi}} \otimes \boldsymbol{n}_{t} \\
& \boldsymbol{W}^{\bar{\eta}}=\boldsymbol{n}_{t} \otimes \boldsymbol{x}^{, \bar{\eta}}-\boldsymbol{x}^{, \bar{\eta}} \otimes \boldsymbol{n}_{t}
\end{aligned}
$$

in a convective basis $\boldsymbol{x}^{\bar{\xi}} \otimes \boldsymbol{x}^{, \bar{\eta}}$, with $\bar{\xi}, \bar{\eta}$ as the contravariant curvilinear coordinates, we obtain the normal change parts of the linearized virtual work as follows:

$$
\begin{aligned}
\delta \Pi_{l i n}^{\Delta n^{g}}= & \int_{\eta^{g}} \int_{\xi^{g}} \frac{p_{t}^{k g}}{2}\left(\begin{array}{c}
\delta \boldsymbol{u}^{g} \\
\delta \boldsymbol{u}_{, \xi}^{g} \\
\delta \boldsymbol{u}_{, \eta}^{g}
\end{array}\right) \cdot\left(\begin{array}{ccc}
\mathbf{0} & \boldsymbol{W}^{\bar{\xi}} & \boldsymbol{W}^{\bar{\eta}} \\
\boldsymbol{W}^{\bar{\xi} T} & \mathbf{0} & \mathbf{0} \\
\boldsymbol{W}^{\bar{\eta} T} & \mathbf{0} & \mathbf{0}
\end{array}\right)\left(\begin{array}{c}
\Delta \boldsymbol{u}^{g} \\
\Delta \boldsymbol{u}_{, \xi}^{g} \\
\Delta \boldsymbol{u}_{, \eta}^{g}
\end{array}\right) d \xi d \eta \\
\delta \Pi_{l i n}^{\Delta n^{f}}= & \int_{\eta^{f}} \int_{\xi^{f}} \frac{p_{t}^{f}}{2}\left(\begin{array}{c}
\delta \boldsymbol{u}^{f} \\
\delta \boldsymbol{u}_{, \xi}^{f} \\
\delta \boldsymbol{u}_{, \eta}^{f}
\end{array}\right) \cdot\left(\begin{array}{ccc}
\mathbf{0} & \boldsymbol{W}^{\bar{\xi}} & \boldsymbol{W}^{\bar{\eta}} \\
\boldsymbol{W}^{\bar{\xi} T} & \mathbf{0} & \mathbf{0} \\
\boldsymbol{W}^{\bar{\eta} T} & \mathbf{0} & \mathbf{0}
\end{array}\right)\left(\begin{array}{c}
\Delta \boldsymbol{u}^{f} \\
\Delta \boldsymbol{u}_{, \xi}^{f} \\
\Delta \boldsymbol{u}_{, \eta}^{f}
\end{array}\right) d \xi d \eta \\
& -\int_{\eta^{f}} \int_{\xi^{f}}\left[p_{t, \xi}^{f}\left(\boldsymbol{x}_{, \eta} \times \delta \boldsymbol{u}_{, \xi}^{f}\right)-p_{t, \eta}^{f}\left(\boldsymbol{x}_{, \xi} \times \delta \boldsymbol{u}_{, \eta}^{f}\right)\right] \cdot \Delta \boldsymbol{u}^{f} d \xi d \eta
\end{aligned}
$$

\subsection{Proof of Conservativeness}

As it was shown in [10] and [5] by product integration and subsequent application of the Gauss theorem the formally unsymmetric part in equation (20) can be transformed into a skew symmetric field term and an unsymmetric boundary term. Assuming physically realistic boundary conditions makes this boundary term to vanish. A complete symmetry of the linearized virtual work $\delta \Pi_{l i n}^{f s i}$ is finally obtained, if the linearized pressure parts (14), (15) and (16) along with the normal change parts (19) and (20) are inserted in the initial equation (13). Further 
sult clearly proves formally the conservativeness of the complete problem, which by general physical consideration is conservative from the origin.

The symmetry of the derived equations will appear clearer after the following FE-discretization.

\subsection{Finite Element Mapping}

After discretizing the linearized virtual fluid structure interaction expression with isoparametric mapping functions $\boldsymbol{N}$ for the displacements, the virtual displacements and the incremental displacements of the structural parts which are in contact with gas/fluid, such that

$$
\boldsymbol{u}=\boldsymbol{N} \boldsymbol{d}, \quad \delta \boldsymbol{u}=\boldsymbol{N} \delta \boldsymbol{d} \quad \text { and } \quad \Delta \boldsymbol{u}=\boldsymbol{N} \Delta \boldsymbol{d},
$$

we obtain from the normal change parts the symmetric load stiffness matrices for each structural element in contact with gas and/or fluid (see also [11], [5], [6], [7], [8]):

$$
\begin{aligned}
\boldsymbol{K}_{\text {elem }}^{g}= & \frac{1}{2} \int_{\eta^{g}} \int_{\xi^{g}} p_{t}^{g}\left(\begin{array}{c}
\boldsymbol{N}_{, \xi} \\
\boldsymbol{N}_{, \xi} \\
\boldsymbol{N}_{, \eta}
\end{array}\right)^{T}\left(\begin{array}{ccc}
\mathbf{0} & \boldsymbol{W}^{\bar{\xi}} & \boldsymbol{W}^{\bar{\eta}} \\
\boldsymbol{W}^{\xi T} & \mathbf{0} & \mathbf{0} \\
\boldsymbol{W}^{\eta \bar{T}} & \mathbf{0} & \mathbf{0}
\end{array}\right)\left(\begin{array}{c}
\boldsymbol{N} \\
\boldsymbol{N}_{, \xi} \\
\boldsymbol{N}_{, \eta}
\end{array}\right) d \xi d \eta, \\
\boldsymbol{K}_{\text {elem }}^{f}= & \frac{1}{2} \int_{\eta^{f}} \int_{\xi^{f}} p_{t}^{f}\left(\begin{array}{c}
\boldsymbol{N} \\
\boldsymbol{N}_{, \xi} \\
\boldsymbol{N}_{, \eta}
\end{array}\right)^{T}\left(\begin{array}{ccc}
\mathbf{0} & \boldsymbol{W}^{\bar{\xi}} & \boldsymbol{W}^{\bar{\eta}} \\
\boldsymbol{W}^{\xi T} & \mathbf{0} & \mathbf{0} \\
\boldsymbol{W}^{\eta \bar{T}} & \mathbf{0} & \mathbf{0}
\end{array}\right)\left(\begin{array}{c}
\boldsymbol{N} \\
\boldsymbol{N}_{, \xi} \\
\boldsymbol{N}_{, \eta}
\end{array}\right) d \xi d \eta \\
& -\frac{\rho_{t}}{2} \int_{\eta^{f}} \int_{\xi^{f}} \boldsymbol{N}^{T}\left(\boldsymbol{n}_{t}^{f} \otimes \boldsymbol{g}+\boldsymbol{g} \otimes \boldsymbol{n}_{t}^{f}\right) \boldsymbol{N} d \xi d \eta .
\end{aligned}
$$

The residual for the corresponding element results in negative right hand side vectors due to fluid and gas pressure loading/support:

$$
\begin{gathered}
\boldsymbol{f}_{\text {elem }}^{g}=-\int_{\eta^{g}} \int_{\xi^{g}} p_{t}^{k g} \boldsymbol{N}^{T} \boldsymbol{n}_{t}^{g} d \xi d \eta \\
\boldsymbol{f}_{\text {elem }}^{f}=\int_{\eta^{f}} \int_{\xi^{f}}\left(-p_{t}^{k g}+p_{t}^{o}-p_{t}^{x}\right) \boldsymbol{N}^{T} \boldsymbol{n}_{t}^{f} d \xi d \eta .
\end{gathered}
$$

Hence along with the coupling vectors reflecting the closed volumes

$$
\begin{gathered}
\boldsymbol{a}_{\text {elem }}=\int_{\eta^{g}} \int_{\xi^{g}} \boldsymbol{N}^{T} \boldsymbol{n}_{t}^{g} d \xi d \eta \quad \text { and } \\
\boldsymbol{b}_{\text {elem }}=\int_{\eta^{f}} \int_{\xi^{f}} \boldsymbol{N}^{T} \boldsymbol{n}_{t}^{f} d \xi d \eta
\end{gathered}
$$

for the elements in contact with gas/fluid and after assembling all local arrays in their corresponding global arrays the linearized state of equilibrium for a closed system partially filled with an incompressible fluid and gas can be written as:

$$
\left(\boldsymbol{K}^{e l}+\boldsymbol{K}^{g}+\boldsymbol{K}^{f}+\alpha_{t}(\boldsymbol{a}+\boldsymbol{b})(\boldsymbol{a}+\boldsymbol{b})^{T}-\gamma_{t} \boldsymbol{b} \boldsymbol{b}^{T}\right) \Delta \boldsymbol{d}=\boldsymbol{f}^{e x}-\boldsymbol{f}^{e l}-\boldsymbol{f}^{f}-\boldsymbol{f}^{g}
$$

In equation (28) the volume dependence of the enclosed gas and fluid is reflected in the rank updates of the stiffness matrix with two dyadic products of the coupling vectors for each closed volume. 
(load-stiffness matrices, coupling vectors and right hand side vectors) have to be set up for each chamber and must be summed up for all $n$ chambers.

$$
\begin{aligned}
\left(\boldsymbol{K}^{e l}+\sum_{i=1}^{n}\left[\boldsymbol{K}_{i}^{g}+\boldsymbol{K}_{i}^{f}+\alpha_{i, t}\left(\boldsymbol{a}_{i}+\boldsymbol{b}_{i}\right)\left(\boldsymbol{a}_{i}+\boldsymbol{b}_{i}\right)^{T}-\gamma_{i, t} \boldsymbol{b}_{i} \boldsymbol{b}_{i}^{T}\right]\right) \Delta \boldsymbol{d} & \\
& =\boldsymbol{f}^{e x}-\boldsymbol{f}^{e l}-\sum_{i=1}^{n}\left[\boldsymbol{f}_{i}^{f}+\boldsymbol{f}_{i}^{g}\right]
\end{aligned}
$$

These multiple rank updates due to volume dependence of the inner state variables of the fluid and gas lead to a fully populated system matrix. However, in order to save memory and computational effort during the solution process it is desirable to have matrices with band structure. Therefore in the next section an algorithm is presented, which benefits both from the dyadic rank updates and the band structure of the decoupled stiffness matrix.

\section{Modal Analysis}

In this section the modal analysis of a fluid/gas supported or loaded structure will be performed. As shown in the previous sections, the volume dependence of the inner state variables leads to several rank updates of the global stiffness matrix $\boldsymbol{K}$. For reasons of simplicity the special case of a single chamber $(i=1)$ only loaded with gas $\left(\boldsymbol{K}^{f}=\mathbf{0}, \boldsymbol{b}=\mathbf{0}, \boldsymbol{f}^{f}=\mathbf{0}\right)$ will serve as an example. The global system matrix $\boldsymbol{A}$ in equation (29) then becomes

$$
\boldsymbol{A}=\boldsymbol{K}^{e l}+\boldsymbol{K}^{g}+\alpha_{t} \boldsymbol{a a ^ { T }}=\boldsymbol{K}+\alpha_{t} \boldsymbol{a a}^{T} .
$$

This dyadic rank update with the coupling vector $\boldsymbol{a}$ results in a fully occupied part of the system matrix $\boldsymbol{A}$, which may lead to numerical difficulties during the modal decomposition process. To bypass the solution with such an almost fully populated system matrix in a first step the standard eigenvalue problem

$$
\boldsymbol{K} \boldsymbol{\psi}_{i}=\lambda_{i} \boldsymbol{\psi}_{i}
$$

has to be solved. With conventional algorithms, as for example subspace iteration method, the eigenvalues $\lambda_{i}$ and the eigenvectors $\boldsymbol{\psi}_{i}$ of the matrix $\boldsymbol{K}$ having the structure of a standard structural matrix can be computed. In the next step the effect of the dyadic update of $\boldsymbol{K}$ on the eigenvalues and -vectors will be investigated.

\subsection{Computation of Shifted Eigenvalues}

Starting from the standard eigenvalue problem

$$
\boldsymbol{A} \boldsymbol{\phi}_{i}=\chi_{i} \phi_{i}
$$

for the volume coupled system matrix $\boldsymbol{A}$, the modal vectors $\phi_{i}$ can be assembled in the modal matrix $\Phi$ and the associated eigenvalues $\chi_{i}$ in the spectral matrix $\boldsymbol{X}$. Rearranging equation (32) then leads to

$$
A \Phi=\Phi X .
$$


All modal vectors are normalized, thus

$$
\boldsymbol{\Psi}^{T} \boldsymbol{\Psi}=\boldsymbol{I} .
$$

Inserting equation (34) in (33) and left hand multiplication with $\Psi^{T}$ yields

$$
\begin{aligned}
\boldsymbol{\Psi}^{T} \cdot \mid \boldsymbol{A} \boldsymbol{\Psi} \boldsymbol{\Xi} & =\boldsymbol{\Psi} \boldsymbol{\Xi} \boldsymbol{X} \\
\boldsymbol{\Psi}^{T} \boldsymbol{A} \boldsymbol{\Psi} \boldsymbol{\Xi} & =\boldsymbol{\Psi}^{T} \boldsymbol{\Psi} \boldsymbol{\Xi} \\
\boldsymbol{\Psi}^{T} \boldsymbol{A} \boldsymbol{\Psi} \boldsymbol{\Xi} & =\boldsymbol{\Xi} \boldsymbol{X}
\end{aligned}
$$

with

$$
\boldsymbol{A}^{\star}=\boldsymbol{\Psi}^{T} \boldsymbol{A} \boldsymbol{\Psi}=\boldsymbol{\Psi}^{T}\left(\boldsymbol{K}+\alpha_{t} \boldsymbol{a a ^ { T }}\right) \boldsymbol{\Psi} .
$$

Thus a modified form of the eigenvalue problem (33) is obtained

$$
\boldsymbol{A}^{\star} \boldsymbol{\Xi}=\boldsymbol{\Xi} \boldsymbol{X}
$$

which can be rewritten for the columns $\boldsymbol{\xi}_{i}$ of the modal factor matrix $\boldsymbol{\Xi}$ and the eigenvalues $\chi_{i}$ :

$$
\boldsymbol{A}^{\star} \boldsymbol{\xi}_{i}=\chi_{i} \boldsymbol{\xi}_{i} .
$$

Substituting (37) into (39) gives us after some reordering

$$
\begin{aligned}
&\left(\boldsymbol{\Psi}^{\boldsymbol{T}}\left[\boldsymbol{K}+\alpha_{t} \boldsymbol{a a ^ { T }}\right] \boldsymbol{\Psi}-\chi_{i} \boldsymbol{I}\right) \boldsymbol{\xi}_{i}=\mathbf{0} \\
&\left(\boldsymbol{\Psi}^{\boldsymbol{T}} \boldsymbol{K} \boldsymbol{\Psi}+\alpha_{t} \boldsymbol{\Psi}^{\boldsymbol{T}} \boldsymbol{a} \boldsymbol{a}^{T} \boldsymbol{\Psi}-\chi_{i} \boldsymbol{I}\right) \boldsymbol{\xi}_{i}=\mathbf{0} .
\end{aligned}
$$

Using the spectral matrix $\Lambda$ of $K$

$$
\Lambda=\Psi^{T} \boldsymbol{K} \Psi
$$

along with the modified coupling vector

$$
\bar{a}=\Psi^{T} a
$$

yields

$$
\left(\boldsymbol{\Lambda}+\alpha_{t} \overline{\boldsymbol{a}} \overline{\boldsymbol{a}}^{T}-\chi_{i} \boldsymbol{I}\right) \boldsymbol{\xi}_{i}=\mathbf{0} .
$$

For this homogeneous set of equation non-trivial solutions $\boldsymbol{\xi}_{i} \neq \mathbf{0}$ do exist, if the determinant of the coefficient matrix $\left(\boldsymbol{\Lambda}+\overline{\boldsymbol{a}} \overline{\boldsymbol{a}}^{T}-\chi_{i} \boldsymbol{I}\right)$ vanishes.

$$
\operatorname{det}\left\{\boldsymbol{\Lambda}+\alpha_{t} \overline{\boldsymbol{a}} \overline{\boldsymbol{a}}^{T}-\chi_{i} \boldsymbol{I}\right\}=0
$$

Now two cases concerning the coordinates $\bar{a}_{j}$ must be considered: 
matrix $\left(\Lambda-\chi_{i} \boldsymbol{I}\right)$ not to be singular. Therefore it can be moved out of the operand in equation (44).

$$
\operatorname{det}\left\{\left(\boldsymbol{\Lambda}-\chi_{i} \boldsymbol{I}\right)\left(\boldsymbol{I}+\alpha_{t}\left[\boldsymbol{\Lambda}-\chi_{i} \boldsymbol{I}\right]^{-1} \overline{\boldsymbol{a}} \overline{\boldsymbol{a}}^{T}\right)\right\}=0
$$

Using the multiplication rule of determinants on equation (45) results in

$$
\operatorname{det}\left(\boldsymbol{\Lambda}-\chi_{i} \boldsymbol{I}\right) \operatorname{det}\left(\boldsymbol{I}+\alpha_{t}\left[\boldsymbol{\Lambda}-\chi_{i} \boldsymbol{I}\right]^{-1} \overline{\boldsymbol{a}} \overline{\boldsymbol{a}}^{T}\right)=0 \text {. }
$$

The determinant of the rank-one-updated identity matrix $\boldsymbol{I}$ can be easily given as

$$
\operatorname{det}\left(\boldsymbol{I}+\alpha_{t}\left[\boldsymbol{\Lambda}-\chi_{i} \boldsymbol{I}\right]^{-1} \overline{\boldsymbol{a}} \overline{\boldsymbol{a}}^{T}\right)=1+\alpha_{t} \overline{\boldsymbol{a}}^{T}\left[\boldsymbol{\Lambda}-\chi_{i} \boldsymbol{I}\right]^{-1} \cdot \overline{\boldsymbol{a}},
$$

see [12]. Thus the following conditional equation for the eigenvalues $\chi_{i}$ is obtained:

$$
\operatorname{det}\left(\boldsymbol{\Lambda}-\chi_{i} \boldsymbol{I}\right)\left(1+\alpha_{t} \overline{\boldsymbol{a}}^{T}\left[\boldsymbol{\Lambda}-\chi_{i} \boldsymbol{I}\right]^{-1} \cdot \overline{\boldsymbol{a}}\right)=0
$$

As the new eigenvalues are different from the old ones, $\chi_{i} \neq \lambda_{i}$, the determinant of the diagonal matrix $\left(\boldsymbol{\Lambda}-\chi_{i} \boldsymbol{I}\right)$ is unequal zero. Consequently the second factor in equation (48) must be identical zero to ensure the existence of non-trivial solutions. For the eigenvalues $\chi_{i}$ the characteristical polynom $p(\chi)$ is obtained:

$$
p(\chi)=1+\alpha_{t} \overline{\boldsymbol{a}}^{T}[\boldsymbol{\Lambda}-\chi \boldsymbol{I}]^{-1} \cdot \overline{\boldsymbol{a}}=0
$$

Because $(\boldsymbol{\Lambda}-\chi \boldsymbol{I})$ is a diagonal matrix, the polynom $p(\chi)$ can be simplified by summing up the product of the reciprocals of the $j^{\text {th }}$ diagonal element with the square of the $j^{\text {th }}$ coordinate $\bar{a}_{j}$.

$$
p(\chi)=1+\alpha_{t} \sum_{j=1}^{n} \frac{\bar{a}_{j} \bar{a}_{j}}{\lambda_{j}-\chi}=0
$$

Function (50) has poles at the eigenvalues $\lambda_{i}$ of the stiffness matrix $\boldsymbol{K}$. Further on function (50) has for a positive $\alpha_{t}$ the following limits between the poles (see also figure 3):

$$
\lim _{\chi \rightarrow \lambda_{j}^{+}}=-\infty, \quad \lim _{\chi \rightarrow \lambda_{j+1}^{-}}=\infty, \quad \lim _{\chi \rightarrow-\infty}=1 \quad \text { and } \quad \lim _{\chi \rightarrow \infty}=1
$$

respectively

$$
\lim _{\chi \rightarrow \lambda_{j}^{+}}=\infty, \quad \lim _{\chi \rightarrow \lambda_{j+1}^{-}}=-\infty, \quad \lim _{\chi \rightarrow-\infty}=1 \text { and } \lim _{\chi \rightarrow \infty}=1,
$$

for a negative $\alpha_{t}$. Besides that, $p(\chi)$ is strictly monotone between its poles, therefore the new eigenvalues $\chi_{i}$ resp. the zero values of $p(\chi)$, must lie somewhere in between the poles. As depicted in figure 3 the new eigenvalues $\chi_{i}$ will increase for a positive pressure volume gradient $\alpha_{t}$, resp. a gas/fluid support.

$$
\lambda_{1}<\chi_{1}<\lambda_{2}<\chi_{2}<\ldots<\lambda_{n}<\chi_{n}
$$

On the other hand a gas/fluid loading $\left(\alpha_{t}<0\right)$ leads to a decrease of the eigenvalues.

$$
\chi_{1}<\lambda_{1}<\chi_{2}<\lambda_{2}<\ldots<\chi_{n}<\lambda_{n}
$$

As already mentioned the polynom $p(\chi)$ is strictly monotone between the poles $\lambda_{j}$, therefore an efficient method to localize the zeroes in $p(\chi)$ can be found by the bisection method. For a given tolerance tol

$$
n>\log _{2}\left(\frac{\lambda_{j+1}-\lambda_{j}}{t o l}\right)
$$

iterations are necessary to approximate the new eigenvalue $\chi_{j}[2]$. 


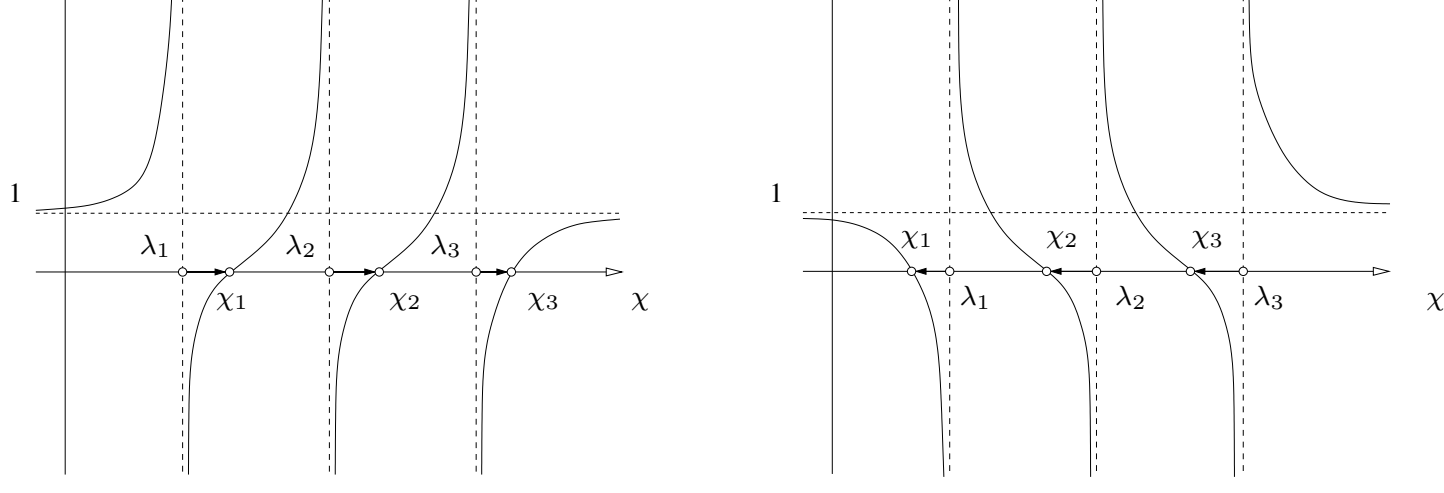

Figure 3: Eigenvalue shift for the example of a 3rd order polynom $p(\chi)$

\subsubsection{Case 2: Coordinate $\bar{a}_{j}=0$}

In this case the matrix

$$
\boldsymbol{\Lambda}+\alpha_{t} \overline{\boldsymbol{a}} \overline{\boldsymbol{a}}^{T}-\chi_{i} \boldsymbol{I}
$$

features only zero entries in the $j^{\text {th }}$ row and the $j^{\text {th }}$ column, except for the diagonal element. This has the effect that the $j^{\text {th }}$ eigenvalue $\chi_{j}$ of (56) equals the old eigenvalue $\lambda_{j}$. Therefore in a first step, all zero entries must be filtered out of the coupling vector $\overline{\boldsymbol{a}}$ and the associated rows and columns in the spectral matrix $\Lambda$ must be reduced likewise. Thus we obtain a reduced coupling vector $\hat{\boldsymbol{a}}$ without any zero entries and a reduced spectral matrix $\hat{\Lambda}$ and can proceed in analogy to case 1 (50) with the characteristical polynom $\hat{p}\left(\chi_{i}\right)$ with a reduced order

$$
\hat{p}\left(\chi_{i}\right)=1+\sum_{j=1}^{m} \frac{\hat{a}_{j} \hat{a}_{j}}{\hat{\lambda}_{j}-\chi}=0, \quad \text { with } \quad \chi \neq \lambda_{j}, \quad m<n .
$$

\subsection{Computation of Eigenvectors}

For the computation of the corresponding eigenvectors equation (43) is considered again, focusing on the column $\boldsymbol{\xi}_{i}$ of the modal factor matrix $\boldsymbol{\Xi}$.

$$
\left(\boldsymbol{\Lambda}+\alpha_{t} \overline{\boldsymbol{a}} \overline{\boldsymbol{a}}^{T}-\chi_{i} \boldsymbol{I}\right) \boldsymbol{\xi}_{i}=\mathbf{0}
$$

Rearranging this equation yields

$$
\begin{aligned}
\left(\boldsymbol{\Lambda}-\chi_{i} \boldsymbol{I}\right) \boldsymbol{\xi}_{i} & =-\alpha_{t}\left(\overline{\boldsymbol{a}} \overline{\boldsymbol{a}}^{T}\right) \boldsymbol{\xi}_{i} \\
\left(\boldsymbol{\Lambda}-\chi_{i} \boldsymbol{I}\right) \boldsymbol{\xi}_{i} & =-\alpha_{t} \overline{\boldsymbol{a}}\left(\overline{\boldsymbol{a}}^{T} \cdot \boldsymbol{\xi}_{i}\right) \\
\boldsymbol{\xi}_{i} & =-\alpha_{t}\left(\boldsymbol{\Lambda}-\chi_{i} \boldsymbol{I}\right)^{-1} \overline{\boldsymbol{a}}\left(\overline{\boldsymbol{a}}^{T} \cdot \boldsymbol{\xi}_{i}\right)
\end{aligned}
$$

To eliminate the implicit form of $\boldsymbol{\xi}_{i}$ it can be normalized by its length, leading to

$$
\begin{aligned}
\boldsymbol{\xi}_{i} & =-\frac{\alpha_{t}\left(\boldsymbol{\Lambda}-\chi_{i} \boldsymbol{I}\right)^{-1} \overline{\boldsymbol{a}}\left(\overline{\boldsymbol{a}}^{T} \cdot \boldsymbol{\xi}_{i}\right)}{\left\|\alpha_{t}\left(\boldsymbol{\Lambda}-\chi_{i} \boldsymbol{I}\right)^{-1} \overline{\boldsymbol{a}}\left(\overline{\boldsymbol{a}}^{T} \cdot \boldsymbol{\xi}_{i}\right)\right\|} \\
& =-\frac{\left(\boldsymbol{\Lambda}-\chi_{i} \boldsymbol{I}\right)^{-1} \overline{\boldsymbol{a}}}{\left\|\left(\boldsymbol{\Lambda}-\chi_{i} \boldsymbol{I}\right)^{-1} \overline{\boldsymbol{a}}\right\|}
\end{aligned}
$$




$$
\boldsymbol{\Phi}=\boldsymbol{\Psi} \boldsymbol{\Xi}=\boldsymbol{\Psi}\left(\begin{array}{cccccc}
\boldsymbol{\xi}_{1} & \boldsymbol{\xi}_{2} & \ldots & \boldsymbol{\xi}_{i} & \ldots & \boldsymbol{\xi}_{n} \\
\mid & \mid & & \mid & & \mid
\end{array}\right)
$$

In the case of zero entries in the coupling vector the reduced modal matrix $\hat{\boldsymbol{\Phi}}$, which can also be computed according to this outlined scheme, must be amended by the old eigenvectors corresponding to the zero entries of $\boldsymbol{a}$ to make it complete.

\subsection{Estimation of accuracy}

In most engineering problems only a few lower eigenvalues are of interest. But on the first sight it may seem that in the above derived algorithm all decoupled eigenvalues $\lambda_{j}$ are needed to set up the characteristical polynom $p(\chi)$ and in addition the whole modal matrix $\Psi$ with all its decoupled eigenvectors is needed to obtain the new modal matrix $\Phi$ with equation (61).

A central factor in the modal analysis within this scheme are the distances of the eigenvalues $\lambda_{i}$ among each other. If all eigenvalues $\lambda_{m}$ are much larger than all previous eigenvalues $\lambda_{k}$, such that

$$
\lambda_{m} \gg \lambda_{k}, \quad \text { with } \quad k=1 . . m-1
$$

yields, all $j$ summands in the characteristical polynom for $m \leq j \leq n$ are negligible compared to the rest. Thus we get - under the assumption $a_{j} \ll \lambda_{m}$, which is usually the case for standard pressures -

$$
\begin{aligned}
p(\chi) & =1+\alpha_{t} \sum_{j=1}^{m-1} \frac{\bar{a}_{j} \bar{a}_{j}}{\lambda_{j}-\chi}+\alpha_{t} \sum_{j=m}^{n} \frac{\bar{a}_{j} \bar{a}_{j}}{\lambda_{j}-\chi}=0 \\
& \approx 1+\alpha_{t} \sum_{j=1}^{m-1} \frac{\bar{a}_{j} \bar{a}_{j}}{\lambda_{j}-\chi}
\end{aligned}
$$

Hence, the bisection method to obtain the zeroes in $p(\chi)$ can be restricted to the search for the lowest eigenvalues. This means that the higher eigenvalues are not affected by the volume dependence as remarkably as the lower ones. Therefore in a first approximation they can remain untouched, which may reduce the computational effort considerably for large FE-problems.

The same argument can be used to reduce the number of eigenvectors $\boldsymbol{\psi}_{i}$ of $\boldsymbol{K}$ necessary for the setup of the new modal matrix $\Phi$. Looking at equation (60) again the non-normalized vector can be written as

$$
\boldsymbol{\xi}_{i}^{\star}=-\left(\boldsymbol{\Lambda}-\chi_{i} \boldsymbol{I}\right)^{-1} \overline{\boldsymbol{a}}=-\left[\begin{array}{c}
a_{1} /\left(\lambda_{1}-\chi_{i}\right) \\
a_{2} /\left(\lambda_{2}-\chi_{i}\right) \\
\vdots \\
a_{n} /\left(\lambda_{n}-\chi_{i}\right)
\end{array}\right]
$$

and its norm is given by

$$
\left|\boldsymbol{\xi}_{i}^{\star}\right|=\sqrt{\left(\frac{a_{1}}{\lambda_{1}-\chi_{i}}\right)^{2}+\left(\frac{a_{2}}{\lambda_{2}-\chi_{i}}\right)^{2}+\ldots+\left(\frac{a_{n}}{\lambda_{n}-\chi_{i}}\right)^{2}} .
$$




$$
\xi_{i j}^{\star}=\frac{a_{j}}{\lambda_{j}-\chi_{i}} \quad \text { for } \quad j<m
$$

respectively

$$
\xi_{i j}^{\star}=0 \text { for } j \geq m .
$$

The length of $\boldsymbol{\xi}_{i}^{\star}$ is less affected by eigenvalues $\lambda_{j}$ with large distance to $\chi_{i}$, because here the squares of the reciprocals of the distances are computed. Thus looking at equation (61)

$$
\boldsymbol{\phi}_{i}=\left(\begin{array}{cccccc}
\mid & \mid & & \mid & & \mid \\
\boldsymbol{\psi}_{1} & \boldsymbol{\psi}_{2} & \ldots & \boldsymbol{\psi}_{j} & \ldots & \boldsymbol{\psi}_{n} \\
\mid & \mid & & \mid & & \mid
\end{array}\right) \boldsymbol{\xi}_{i}
$$

follows that the $j^{\text {th }}$ coordinate in vector $\boldsymbol{\xi}_{i}$ obviously represents the influence of the old modal vector $\boldsymbol{\psi}_{j}$ of $\boldsymbol{K}$ on the computation of the shifted eigenvector $\phi_{i}$ due to the rank-1-update. Hence to estimate the accuracy of the eigenmodes $\phi_{i}$ computed with a reduced set of $m$ eigenvalues $\lambda_{j}$ and -vectors $\boldsymbol{\psi}_{j}$ the modal factor matrix $\boldsymbol{\Xi}$ must be considered. In the case of a fully populated matrix the mutual influence of the eigenvectors is obviously high, which means that a new computation with a larger set of eigenvalues $\lambda_{j}$ and -vectors $\boldsymbol{\psi}_{j}$ is probably necessary. In the case of a less populated modal factor matrix with a dominantly diagonal shape, a new computation with a larger modal set would not affect the solution remarkably.

\subsection{Multiple Rank updates}

Although it may seem that the derived algorithm is only valid for a rank-one-update of the stiffness matrix $\boldsymbol{K}$, multiple rank updates can easily be taken into account by a subsequent application of this method. I.e. in the case of a closed chamber, which is partially filled with a heavy incompressible fluid and an additional gas volume, according to equation (28) the system matrix $\boldsymbol{A}$ is obtained by a rank-2-update of $\boldsymbol{K}$ :

$$
\boldsymbol{A}=\boldsymbol{K}+\alpha_{t}(\boldsymbol{a}+\boldsymbol{b})(\boldsymbol{a}+\boldsymbol{b})^{T}-\gamma_{t} \boldsymbol{b} \boldsymbol{b}^{T}
$$

To compute the eigenvalues and -vectors the stiffness matrix with the first rank update is split from (69), which leads to a matrix $\boldsymbol{A}^{1}$ only affected by the gas volume change $(\boldsymbol{a}+\boldsymbol{b})$ :

$$
\boldsymbol{A}^{1}=\boldsymbol{K}+\alpha_{t}(\boldsymbol{a}+\boldsymbol{b})(\boldsymbol{a}+\boldsymbol{b})^{T}
$$

For this rank-one-update the eigenvalues and -vectors can be computed as described in the previous section. In the same manner the shift of eigenvalues and -vectors of $\boldsymbol{A}^{1}$ due to the second rank update can be computed.

$$
\boldsymbol{A}^{2}=\boldsymbol{A}^{1}-\gamma_{t} \boldsymbol{b} \boldsymbol{b}^{T}
$$

\section{Numerical Examples}

In the previous sections the algorithms for the computation of structures under hydrostatic loading were derived. The numerical examples are chosen to show the effect of the volume terms on the stability behavior of deformable structures. 
gas pressure $p_{0}$ acting in an initial volume $v_{0}$. I he loading will be siowiy increased and atter equilibrium has been found in the current load step the eigenvalues will be computed. In order to emphasize the volume dependence the arch was modelled with a very soft material: Young's modulus $E=60 \mathrm{~N} / \mathrm{mm}^{2}$, Poisson ratio $\nu=0.4$. This corresponds with the material data of a fiber reinforced rubber membrane, which finds its application in the field of inflatable dams. Especially such gas supported dams suffer offen from instability at certain upstream fluid levels.

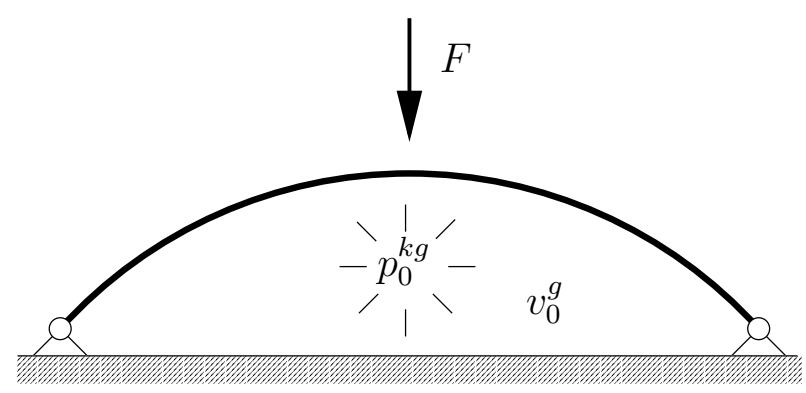

Figure 4: Arch under single load

Figure 5 shows the development of the first 4 eigenvalues $\lambda_{i}, i=1 . .4$ (relative to their initial value) during the loading process for a system without any volume coupling and normal change parts (no FSI) and thus considering the internal pressure as an internal load only and for a system considering the volume change and the normal change in the eigenvalue computation (FSI).

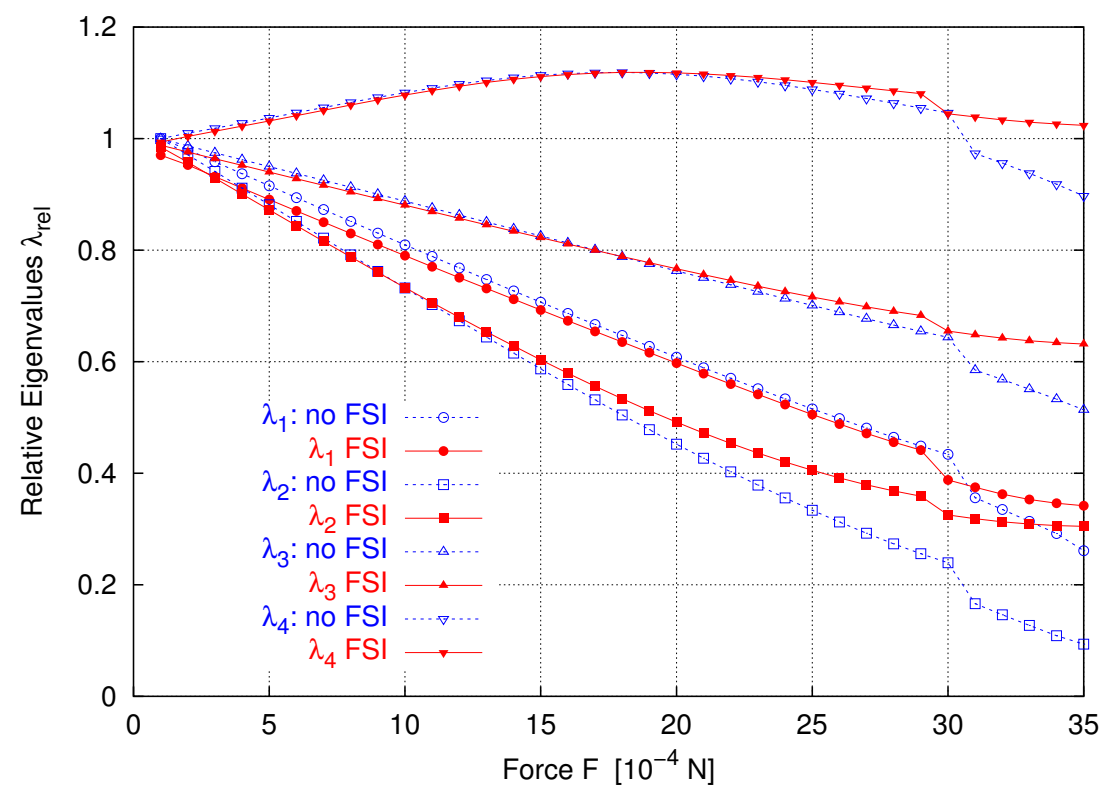

Figure 5: Development of eigenvalues over external loading

At the beginning in both analyses the first three eigenvalues decrease almost linearly with in- 
quickly, whereas the eigenvalues of the volume coupled model only decrease very slowly. As expected the structure shows some stiffening if the volume dependence is taken into account.

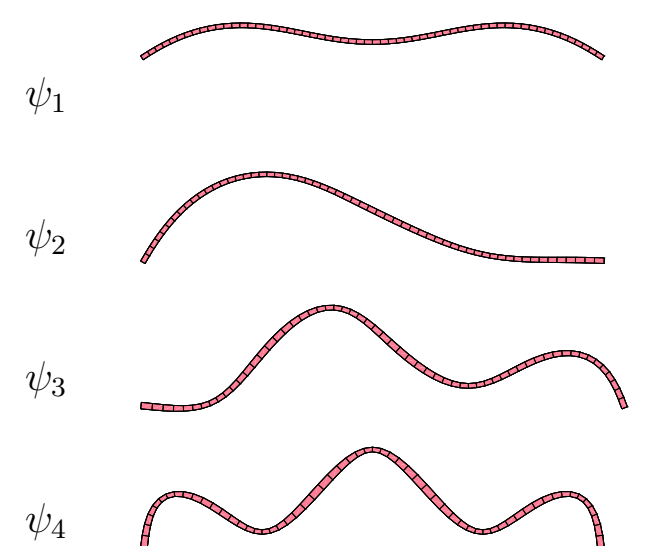

Figure 6: Eigenmodes $\psi_{i}$ without volume dependence (load step $F=35 \cdot 10^{-4} N$ )

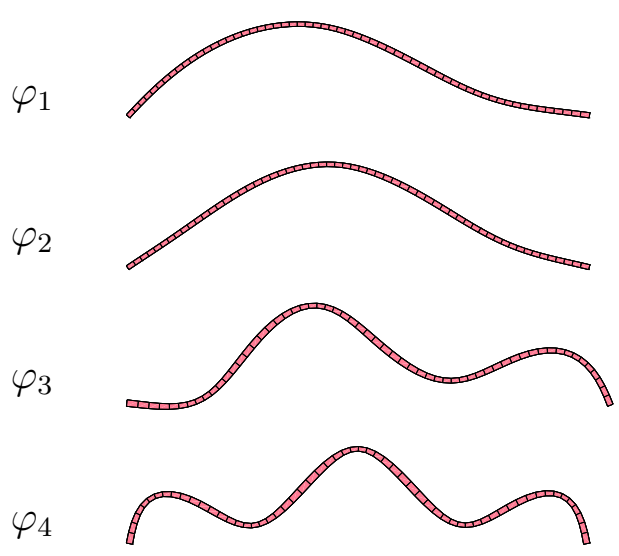

Figure 7: Eigenmodes $\varphi_{i}$ with volume dependence (load step $F=35 \cdot 10^{-4} N$ )

Considering the volume dependence (figure 7) the first two eigenmodes $\varphi_{1}$ and $\varphi_{2}$ differ stronlgy from the eigenmodes $\psi_{1}$ and $\psi_{2}$ in figure 6 taking into account only loading, whereas the subsequent modes are almost identical. Figure 8 gives the reason for the new shape of the altered eigenmodes $\varphi_{i}$. It displays the coordinates of the modal factor matrix $\boldsymbol{\Xi}$. The coordinate $\Xi_{j i}$ characterizes the influence of the of old eigenmodes $\psi_{j}$ on the new eigenmodes $\varphi_{i}$ and thus represents some kind of filter. The first eigenvalue $\varphi_{1}$ depends by a factor $\Xi_{11}=.97$ on the eigenvalue $\psi_{1}$ and by a factor $\Xi_{21}=.22$ on the eigenvalue $\psi_{2}$. The higher eigenmodes have almost no influence on $\varphi_{1}$. The same is true for the second eigenmode $\varphi_{2}$, which mainly depends on $\psi_{1}$ and $\psi_{2}$. Each of the higher eigenmodes $\varphi_{3}$ resp. $\varphi_{4}$ is only affected by its counterpart $\psi_{3}$ (with a factor $\Xi_{33} \approx 1.0$ ) respectively $\psi_{4}$ (with $\Xi_{44} \approx 1.0$ ).

\subsection{Gas supported steel cylinder under axial loading}

Now the effect of the gas support will be investigated at the example of a gas filled ( $p_{0}^{k g}=$ $10^{-2} \mathrm{~N} / \mathrm{mm}^{2}$ ) steel cylinder under axial loading (height $h=8 \mathrm{~m}$, radius $r=2 \mathrm{~m}$ and thickness $t=r / 1000$ ), which is clamped at both sides. For this purpose the axial loading on the cylinder acting as a line load on the upper edge is slowly increased and after equilibrium has been found for each load step, the eigenvalues $\lambda_{i}$ and the associated eigenvectors $\varphi_{i}$ of the stiffness matrix will be computed, both for a full FSI-support (considering the volume change and the normal change) and without FSI-support (considering the internal pressure as a pure loading). The effect of the gas on the longitudinal loading and support is not taken into account.

Figure 9 displays the development of the first eigenvalue over the internal gas pressure loading. For both analyses with an increasing axial load the eigenvalue almost linearly decreases until load step 15. Here a formerly higher eigenmode becomes the new eigenmode associated to the lowest eigenvalue. The same can be observed at load step 25, where again a formerly higher eigenmode becomes the lowest one. From this point on the stiffness of the steel cylinder 


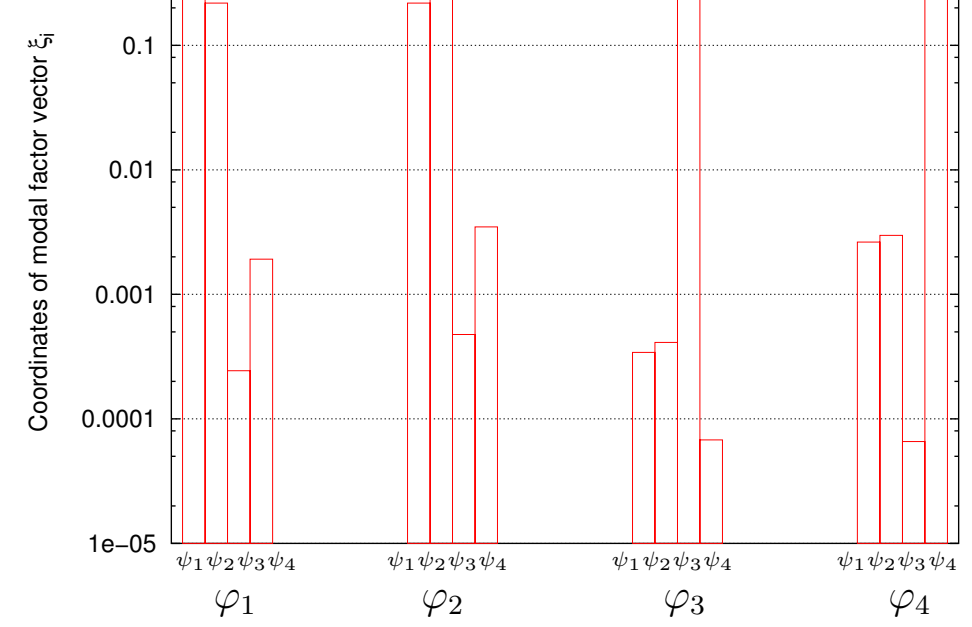

Figure 8: Influence of old eigenmodes $\psi_{i}$ on new eigenmodes $\varphi_{i}\left(\operatorname{load}\right.$ step $\left.F=35 \cdot 10^{-4} \mathrm{~N}\right)$

decreases with a very high rate. Figures 10 and 11 show the first and the fourth eigenmode at the beginning of the axial loading and in the vicinity of the buckling load. Because of the mode switching during the loading process the former first eigenmode $\varphi_{1}$ becomes $\varphi_{4}$ at the end. Further on from figure 9 we can conclude that the structure computed with FSI-support behaves slightly stiffer at the beginning and after the second mode becomes the first one it loses its stiffness faster than the model considering the pressure as a pure loading. The deformation of the structure leads to an increased volume and thus the internal pretension caused by the gas pressure is reduced. On the other hand the stiffening effect of the gas - in this example appears to be too small to compensate this loss of pretension and thus the stiffness declines with continuing deformation.

This example also shows the importance of the consideration of the volume dependence of the internal state variables (here the gas pressure). Although there is almost no influence on the eigenmodes (modal factor matrix $\boldsymbol{\Xi}$ is almost identical to the identity matrix), the volume dependence remarkably affects the stiffness of the structure in the vicinity of the buckling load. The computation without FSI-support thus may lead to an overestimation the critical buckling load.

\section{Conclusions}

This contribution presented some investigation of the influence of gas or fluid support on the eigenvalues and eigenmodes of the stiffness matrix of shell or membrane-like structures undergoing large displacements. The derived equations considered the change of the inner state variables (e.g. the gas pressure $p^{k g}$ ) during the deformation process depending on volumetric effects as e.g. a closed containment. This led to a stiffness matrix, with additional dyadic updates, which allowed the derivation of an efficient algorithm to compute the eigenvalues and eigenmodes just by shifting the old eigenvalues. The effect of the gas or fluid support is reflected in the coordinates of the modal factor matrix: For a nearly unaffected system (as in the case of the gas supported steel cylinder) it has approximatly the shape of the identity matrix. In the 


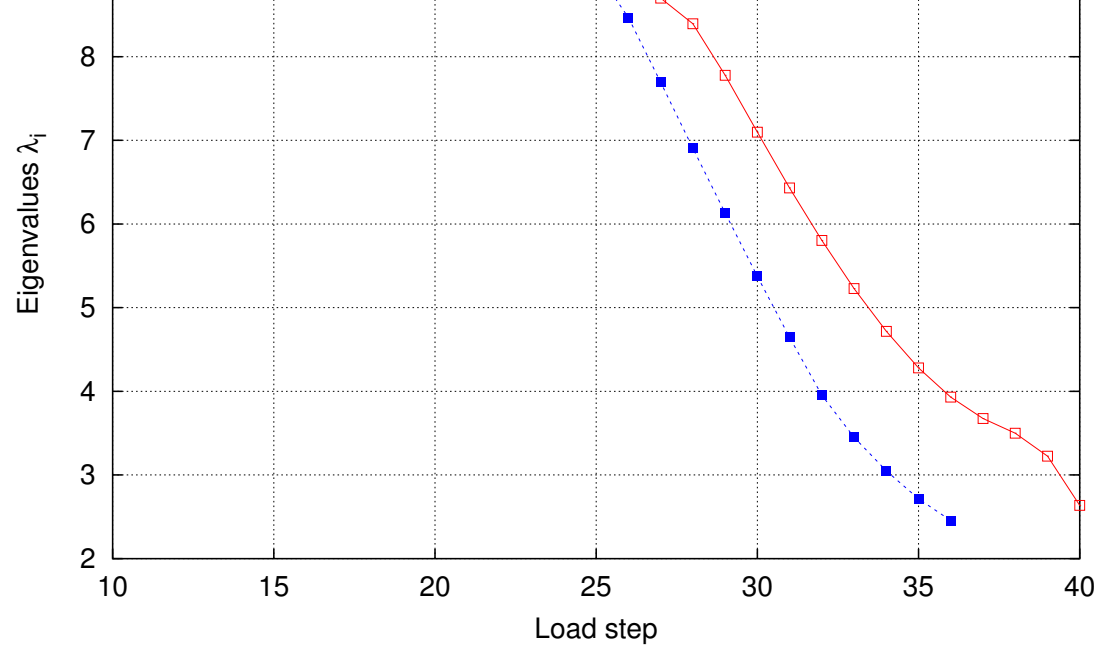

Figure 9: Development of first eigenvalue over loading time

case of the soft membrane structure, it was fully occupied by non-zero entries, which led to eigenmodes differing considerably to the original modes.

The examples showed that for a modal analysis in the case of soft structures a consideration of the volume dependence of the gas/fluid support is important, because the structure is gaining a certain amount of additional stiffness. For stiffer structures however, the volume dependence is also not necessarily negligible. The computation of the eigenvalues with FSI-support led to smaller values than without FSI-support for the particular example of a gas supported cylinder under axial load.

\section{REFERENCES}

[1] J. Bonet, R. D. Wood, J. Mahaney, P. Heywood, Finite element analysis of air supported membrane structures. Computer Methods in Applied Mechanics and Engineering, 190, 579-595, 2000.

[2] J. D. Faires and R. L. Burden, Numerische Methoden. Spektrum Akademischer Verlag, 1994.

[3] R.H. Luchsinger and A. Pedretti and A. Steingruber and M. Pedretti, The new structural concept Tensairity: Basic principles. Proceedings of the Second Conference of Structural Engineering, Mechanics and Computation, A.A. Balkema/Swets Zeitlinger, Lisse, 2004.

[4] M. Haßler and K. Schweizerhof, On the Interaction of Fluid and Gas Loaded MultiChamber Systems in a Large Deformation Finite Element Analysis (submitted for publication). 2006.

[5] K. Knebel, Stabilität von Stahlzylindern mit unilateralen Randbedingungen bei statischen und dynamischen Beanspruchungen. PhD-thesis, Institut für Mechanik der Universität Karlsruhe (TH), 1997 (in German). 


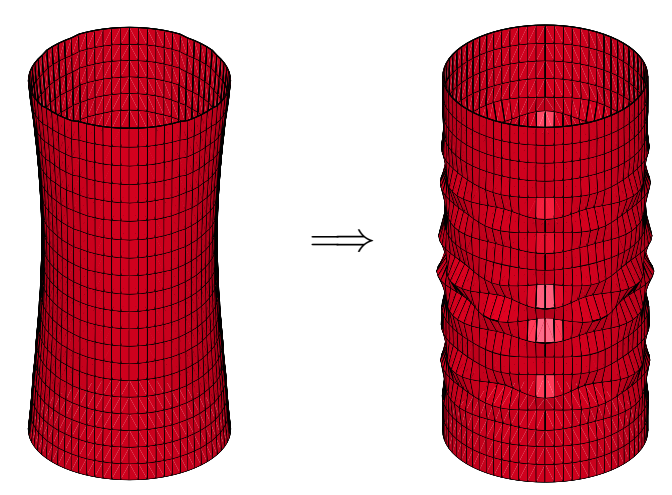

Figure 10: First eigenmode $\varphi_{1}$ at the beginning of loading (a) and in vicinity of buckling load (b)

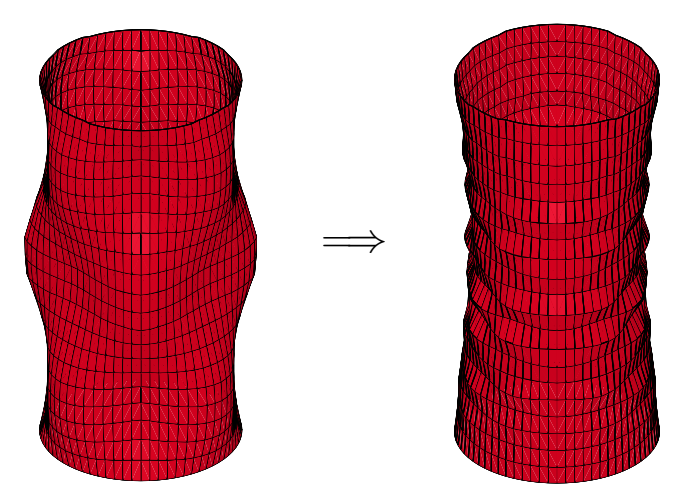

Figure 11: Fourth eigenmode $\varphi_{4}$ at the beginning of loading (a) and in vicinity of buckling load (b)

[6] T. Rumpel, Effiziente Diskretisierung von statischen Fluid-Struktur-Problemen bei großen Deformationen. PhD-thesis, Institut für Mechanik der Universität Karlsruhe (TH), 2003 (in German).

[7] T. Rumpel and K. Schweizerhof, Volume-dependent pressure loading and its influence on the stability of structures. International Journal for Numerical Methods in Engineering, 56, 211-238, 2003.

[8] T. Rumpel and K. Schweizerhof, Hydrostatic fluid loading in non-linear finite element analysis. International Journal for Numerical Methods in Engineering, 59, 849-870, 2004.

[9] T. Rumpel, K. Schweizerhof and M. Haßler, Efficient Finite Element Modelling and Simulation of Gas and Fluid Supported Membrane and Shell Structures. Recent Advances in Textile Membranes and Inflatable Structures, E. Onate, B. Kröplin (eds.), 2004.

[10] K. Schweizerhof, Nichtlineare Berechnung von Tragwerken unter verformungsabhängiger Belastung. PhD-thesis, Institut für Baustatik der Universität Stuttgart, 1982 (in German).

[11] K. Schweizerhof and E. Ramm, Displacemant dependent pressure loads in non-linear finite element analyses. Computers \& Structures, 18, 1099-1114, 1984.

[12] K. Schweizerhof, Quasi-Newton Verfahren und Kurvenverfolgungsalgorithmen für die Lösung nichtlinearer Gleichungssysteme in der Strukturmechanik. Institut für Baustatik der Universität Karlsruhe (TH), 1989 (in German).

[13] P. Wriggers, Nichtlineare Finite-Element-Methoden. Springer-Verlag Berlin, 2001.

[14] H. Ziegler, An introduction to thermomechanics. North-Holland Publishing Company, 1977.

[15] O. C. Zienkiewicz and R. L. Taylor, The finite element method, Vol. 1. ButterwothHeinemann, 2000. 